\title{
Diospyros kaki: Kaki oder Kakipflaume
}

\author{
Ingo Hetzel \& Armin Jagel
}

\begin{abstract}
Persimmon (Diospyros kaki) fruits of different varieties have conquered our fruit counters for several years. When fully ripe, the orange-coloured fruits taste juicy-sweet. In the Palmengarten and, generally, in mild areas of Germany, the species is hardy and sets fruit when planted outside. Morphology, ecology and use are presented. In addition, Diospyros lotus and Diospyros virginiana are described. These two wild species have significantly smaller but equally edible fruits. However, they have no economic significance.
\end{abstract}

\section{Zusammenfassung}

Kakifrüchte (Diospyros kaki) verschiedener Sorten haben seit einigen Jahren unsere Obsttheken erobert. Bei Vollreife schmecken die orange gefärbten Früchte saftig-süß. In milden Gebieten Deutschlands, so auch im Palmengarten, ist die Art winterhart und trägt, wenn sie ausgepflanzt ist, reichlich Früchte. Morphologie, Ökologie und Verwendung werden vorgestellt. Zusätzlich werden Diospyros lotus und Diospyros virginiana beschrieben. Diese beiden Wildarten haben deutlich kleinere, aber ebenso essbare Früchte. Sie haben aber keine wirtschaftliche Bedeutung.

\section{Einleitung}

Besonders im Winterhalbjahr, wenn die Erntezeit heimischer Früchte vorüber ist, bereichern immer mal wieder neue, weniger bekannte Südfrüchte das Obstsortiment der hiesigen Supermärkte, Feinkostläden und (Weihnachts-)Märkte. Seit einigen Jahren gibt es bei uns regelmäßig die sogenannten Kakis (auch Kakipflaumen genannt, Diospyros kaki) zu kaufen. Bei dem Namen stellt sich eher eine Assoziation in Richtung eines grün-braunen bzw. erdartigen Farbtons von Armeeuniformen oder Tropenkleidung ein. Ka-

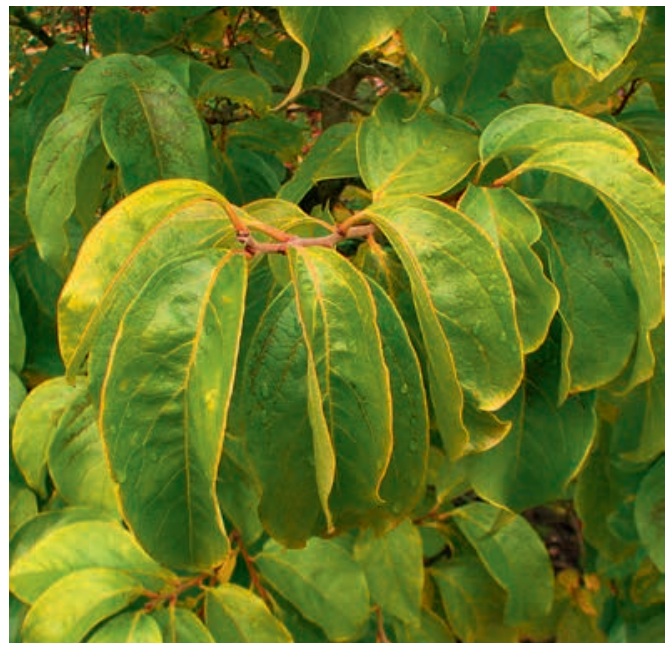

Abb. 1: Belaubter Zweig von Diospyros kaki.

(Foto: V. M. Doerken) kifrüchte sind aber keineswegs „khaki“-farbig, sondern auffällig orange bis rot gefärbt. Der Name leitet sich vom japanischen Namen der Frucht ab.

Der Kakibaum (Diospyros kaki, engl. Japanese Persimmon) gehört zu den Ebenholzgewächsen (Ebenaceae), ist somit mit dem tropischen Baum, der das wertvolle schwarze Kernholz liefert (z. B. Diospyros ebenum, Ceylon-Ebenholzbaum), eng verwandt. Die aus zwei Gattungen mit etwa 500600 Arten bestehende Familie ist vorwiegend in

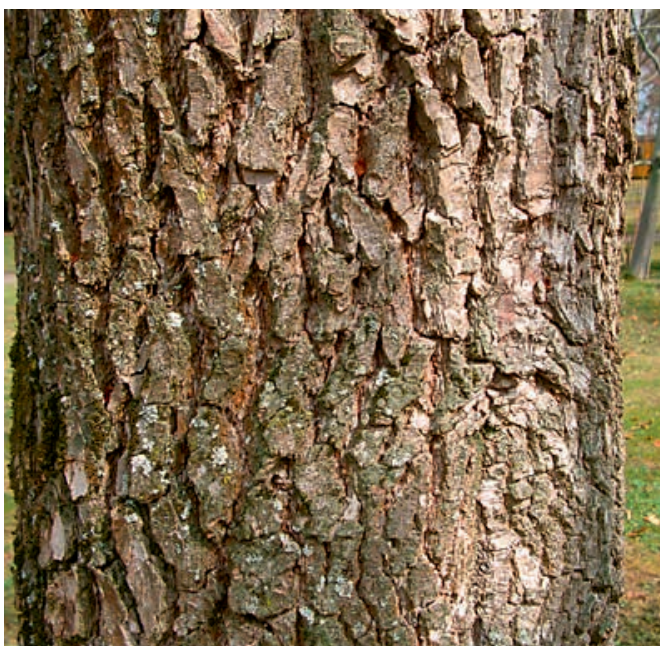

Abb. 2: Stamm von Diospyros kaki.

(Foto: V. M. Doerken) 


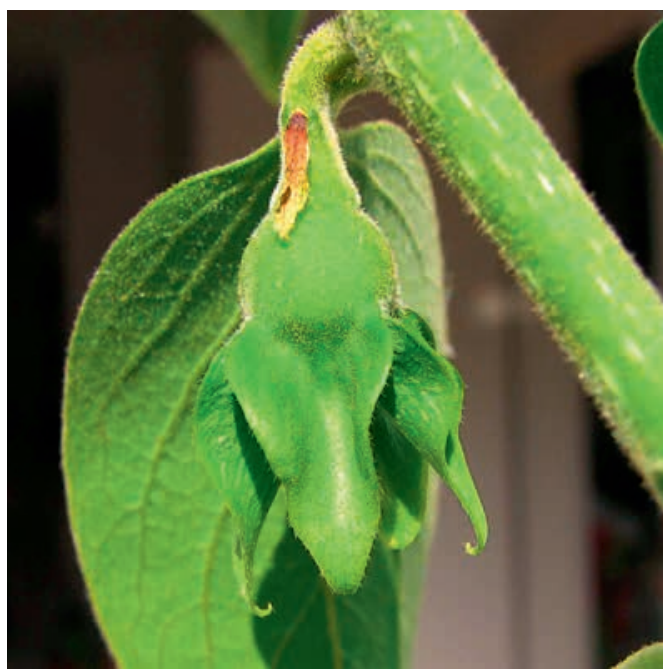

Abb. 3: Blüte von Diospyros kaki. (Foto: A. JAGEL)

tropisch-subtropischen Gebieten verbreitet und hat ihren Schwerpunkt im südöstlichen Asien, während sie in Australien und Südamerika nur schwach vertreten ist (Wallnöfer 2004). Der bis $15 \mathrm{~m}$ hohe Kakibaum ist ursprünglich in China beheimatet, wo nach Angaben Marco Polos mit den Früchten bereits im frühen 14. Jahrhundert Handel getrieben wurde. Die Art wurde außerdem schon früh nach Japan gebracht, wo sie sich noch heute großer Beliebtheit erfreut. Diospyros kaki wird mittlerweile weltweit in den Subtropen als Obstbaum angebaut, die wichtigsten Anbaugebiete sind Spanien, Italien, Israel, Japan, Neuseeland, Florida und Kalifornien (Trueb 1999, LyLE 2006).

In Europa wird der Kakibaum der wintermilden Härtezone $8 \mathrm{~b}$ zugeordnet, also einem Bereich, in dem er bei jährlichen Minimumtemperaturen von $-9,4$ bis $-6,7^{\circ} \mathrm{C}$ noch winterhart ist (Heinze \& Schreiber 1984). Diese Winterhärtezone tritt in Mitteleuropa in wintermilden Bereichen auch an der deutsch-niederländischen Nordseeküste und auf den dänischen Inseln auf. Auch im Rhein-Main-Gebiet ist der Kakibaum winterhart. Ein reichlich fruchtendes Exemplar ist im Palmengarten in der Nähe des Betriebshofes neben dem Oktogonhäuschen mit kalifornischer Bepflanzung zu bewundern. Auch im Botanischen

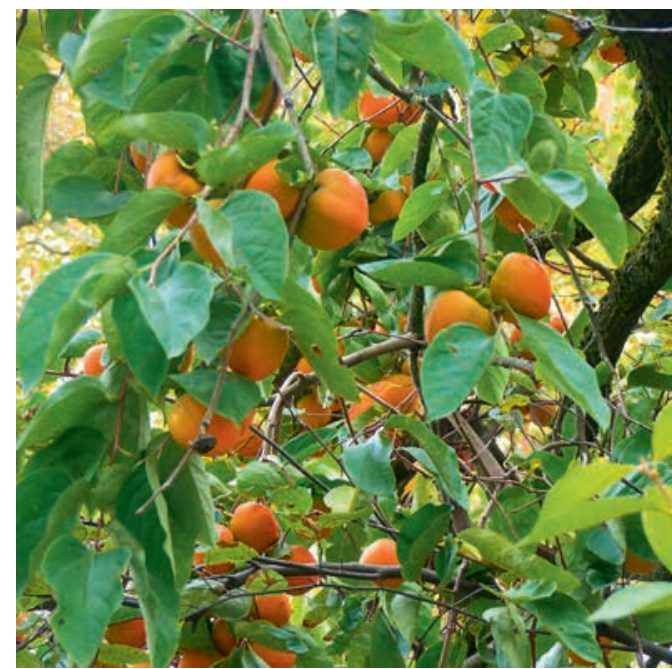

Abb. 4: Üppig fruchtender Kakibaum im Palmengarten. (Foto: H. STEINeCKe)

Garten Mainz fruchten zwei ausgepflanzte Exemplare regelmäßig. In Düsseldorf wächst ein Kakibaum seit Jahren im Botanischen Garten an einer geschützten Stelle und bildet ebenso Früchte aus. Im Ruhrgebiet wird er meist als Kübelpflanze gehalten, an milden Standorten kann er auch hier ausgepflanzt werden.

\section{Name}

Der von CARL von Linné eingeführte Gattungsname Diospyros bezog sich zunächst nur auf die beiden verwandten Arten $D$. lotus und $D$. virginiana und wurde erst später auf $D$. kaki übertragen. Er leitet sich aus dem Griechischen ab und bedeutet „Götterspeise“. Die aus dem Englischen stammende Bezeichnung „Persimmon“ (im Deutschen „Persimone“) stammt aus der Sprache der Algonquin, einem aus mehreren Stämmen bestehenden Volk nordamerikanischer Ureinwohner. Der Name „Kaki“ ist die Kurzform des japanischen Namens „Kakinoki“ für die Kulturform bzw. „Yamakaki“ für die Wildform (Genaust 2005, LyLE 2006).

\section{Blätter und Blüte}

Die Blätter des Kakibaumes sind eiförmig bis länglich und können $20 \mathrm{~cm}$ lang werden. Sie sind oberseits dunkelgrün glänzend und unterseits heller blaugrün gefärbt. Im Herbst zeichnen sie sich 


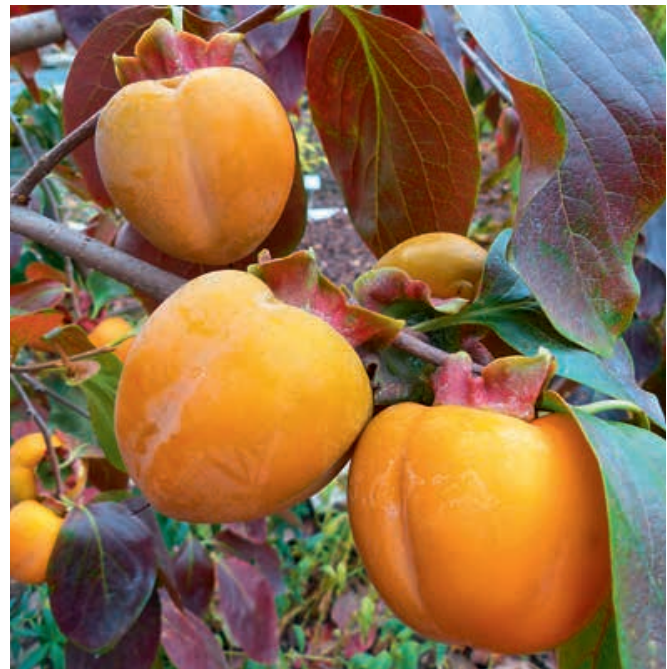

Abb. 5: Zweig mit Kakifrüchten und rötlichem Herbstlaub im Botanischen Garten Mainz. (Foto: H. STeinecke)

durch eine intensive gelbe bis orangerote Herbstfärbung aus.

Die grünlich gelben, etwa $1-1,5 \mathrm{~cm}$ großen Kakiblüten öffnen sich in Deutschland meist im Juni. Die Zuchtformen zeichnen sich dabei durch eine starke Variabilität der Blütenorgane aus. So gibt es Sorten, die ausschließlich weibliche Blüten tragen, andere haben neben weiblichen auch zweigeschlechtliche Blüten, wieder andere tragen Blüten beider Geschlechter (Trueb 1999, Lyle 2006). Nach der Blütezeit bleibt der massive grüne Kelch erhalten (Abb. 3, 5).

\section{Früchte und ihre Sorten}

Ein einziger Kakibaum kann als Obstbaum im günstigsten Fall jährlich bis zu 100 kg Früchte liefern (Nowak \& Schulz 1998). Die Kakifrucht ist morphologisch betrachtet eine Beere mit vier bis acht Samen. Bei den Zuchtformen, die bei uns verkauft werden, wurden die Samen allerdings weggezüchtet und sind nur noch vereinzelt zu finden. An den Stellen, wo ursprünglich die Samen saßen, erkennt man im Querschnitt noch die leeren Fruchtkammern (Abb. 9).

Früchte der Zuchtformen werden etwa $12 \mathrm{~cm}$ lang. Die Farbe der Früchte variiert je nach Sorte und Reifezustand zwischen gelborange bis orange-

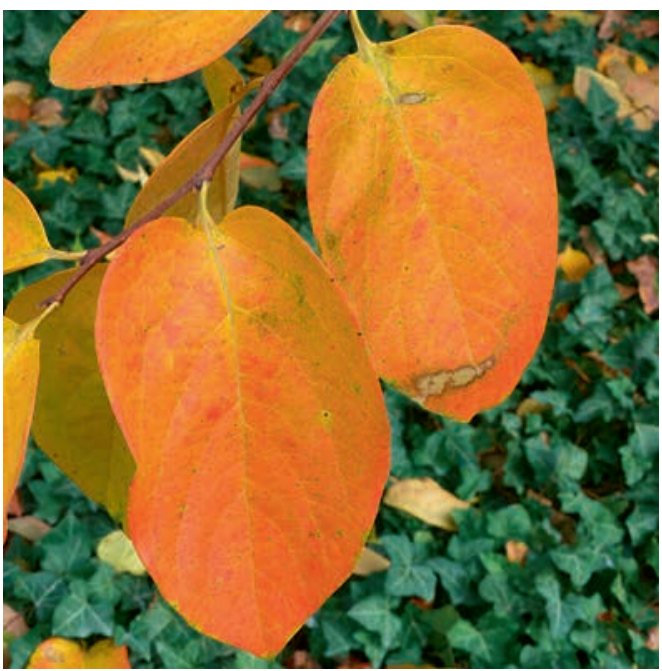

Abb. 6: Leuchtend orange gefärbtes Herbstlaub eines Kakibaumes im Palmengarten. (Foto: H. STEInecke)

rot. Außerdem können sie verschiedene Formen aufweisen.

Aufgrund ihres hohen Gehaltes an Tannin, einem pflanzlichen Gerbstoff, sind Kakis erst in vollreifem Zustand wohlschmeckend. Das sogenannte Kaki-Tannin ist für die adstringierende (zusammenziehende) Wirkung und ein unangenehm raues, pelziges Gefühl im Mund verantwortlich. Erst wenn die Früchte voll- oder sogar überreif sind, lösen sie diese Empfindung nicht mehr aus. Auch durch eine 24-stündige Lagerung

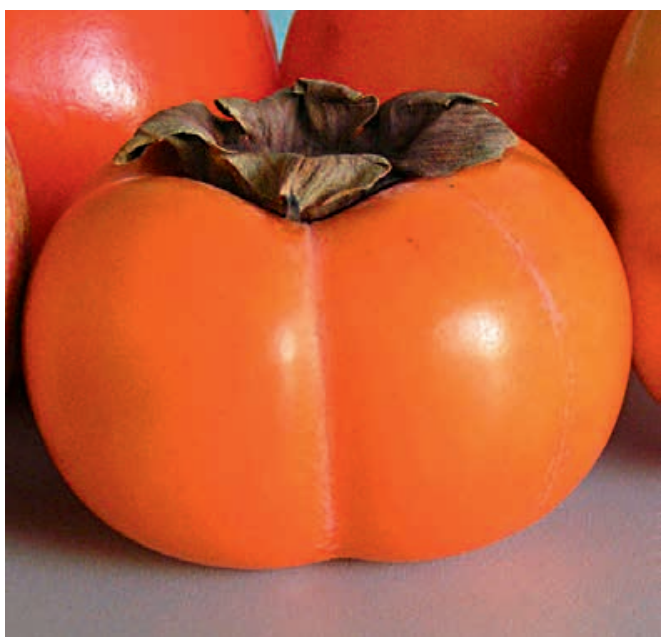

Abb. 7: Handelsübliche Sharonfrucht. (Foto: I. Hetzel) 


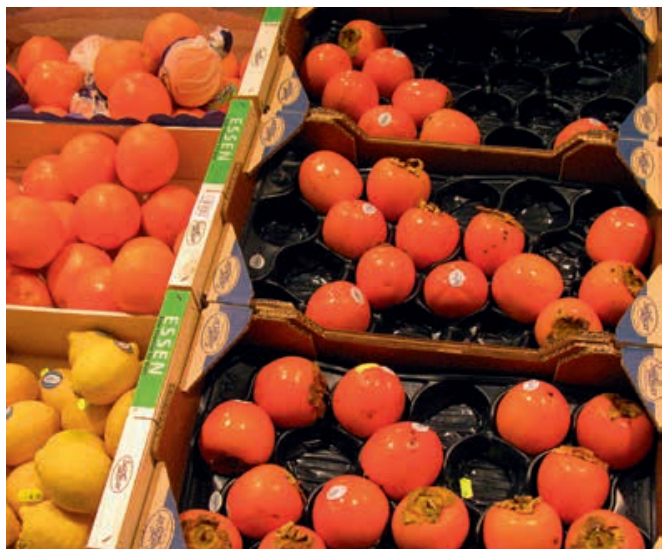

Abb. 8: Kakifrüchte im Angebot eines Supermarktes.

(Foto: A. JAGEL)

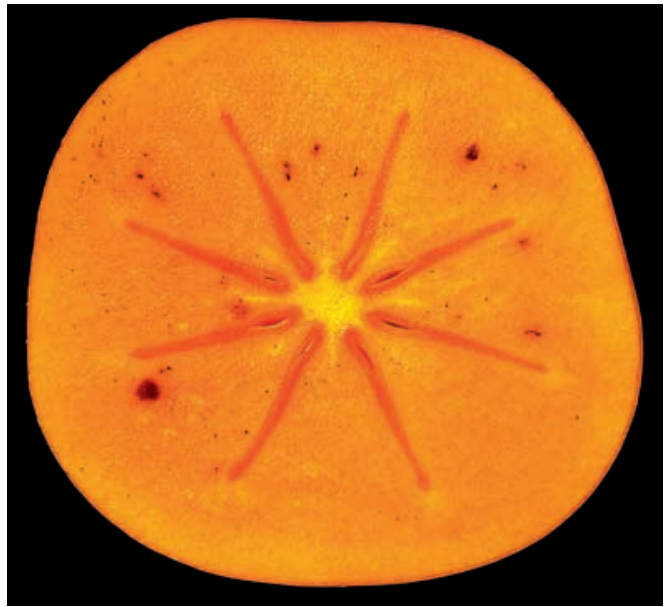

Abb. 9: Querschnitt durch die Frucht von Diospyros kaki 'Triumph'. Die spaltenförmigen Fruchtfächer enthalten keine Samen. (Foto: J. MarkwirTh)

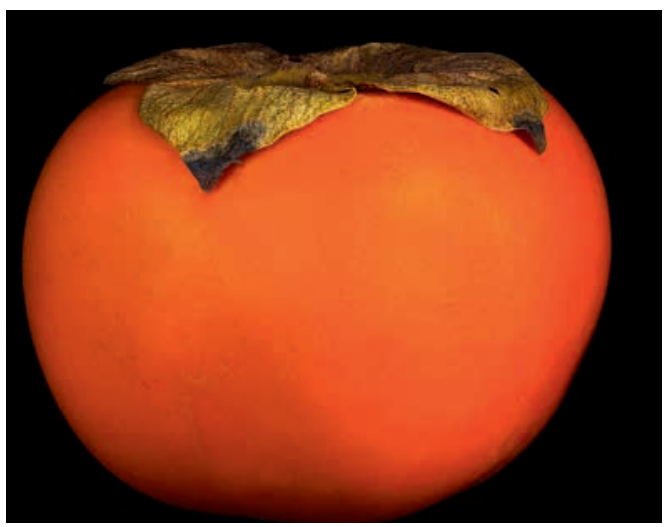

Abb. 10: Ganze Frucht von Diospyros kaki 'Triumph'. (Foto: J. MARKWIRTH)

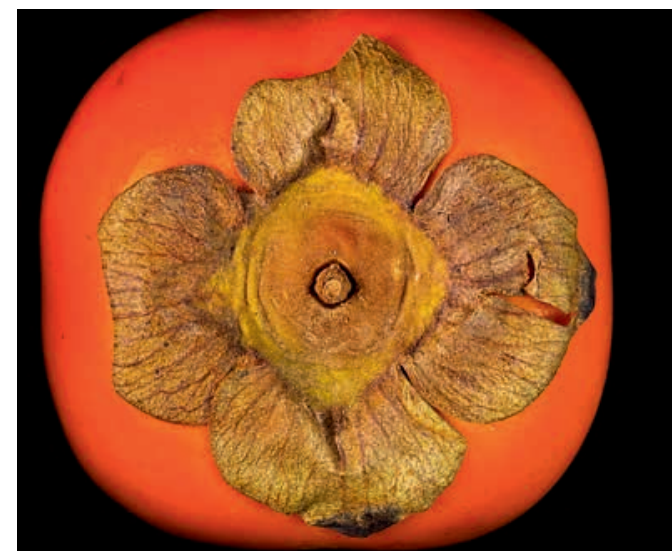

Abb. 11: Aufsicht auf die Frucht von Diospyros kaki 'Triumph' mit Kelch. (Foto: J. Markwirth)

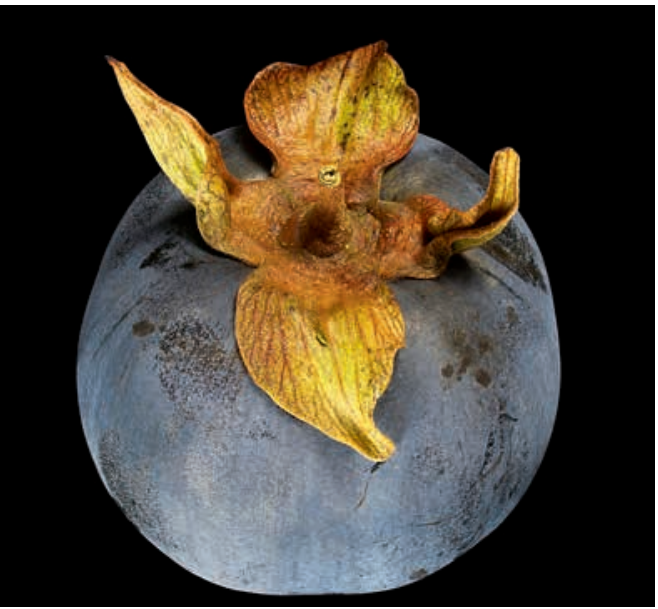

Abb. 12: Diospyros kaki var. silvestris, Chinesische Persimone, nach Frosteinwirkung aus dem Botanischen Garten Frankfurt. (Foto: J. MARKWIRTH)

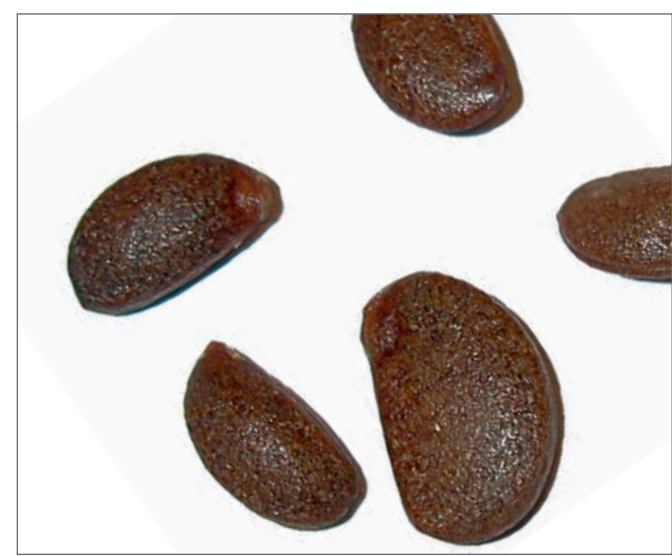

Abb. 13: Samen aus einer Kakifrucht. (Foto: A. JAGEL) 
in einer Gefriertruhe sollen die Tannine abgebaut werden (LyLE 1977).

Heute werden in Deutschland von Mitte Oktober bis Mitte Dezember überwiegend große, längliche Früchte angeboten, die im Obsthandel als Kaki bezeichnet werden und hinter denen sich insbesondere die Sorten 'Tipo', 'Loto di Romagna' (beide aus Italien) oder 'Rojo Brillante' (aus Spanien) verbergen. Daneben gibt es eine deutlich unterschiedlich geformte, fleischtomatenförmig abgeflachte Sorte, die im Sharontal in Israel gezüchtet wurde und danach ihren Namen "Sharon" bekam. Diese Kaki-Sorte hat auch im Deutschen den Namen übernommen und wird manchmal darüber hinaus als Sharoni oder Sharon-Frucht bezeichnet. Bei ihr ist der Gerbstoffgehalt stark reduziert, wodurch sie bereits im festeren (unreiferen) Zustand genießbar und länger haltbar sind.

Weltweit betrachtet ist die Formenvielfalt sehr viel reicher und unübersichtlicher. So sind heute mehr als 2000 verschiedene Sorten bekannt, die sich bezüglich Größe, Farbe und vor allem im Geschmack unterscheiden. Die Anzahl der Sorten nimmt dabei beständig zu, da immer mehr Sorten miteinander gekreuzt werden (TRUEB 1999, LyLE 2006). Eine Übersicht über einige Sorten gibt z. B. die Internetseite www.kaki-info.de.

\section{Verwendung}

Kakis werden als Obst ungeschält oder mit Schale gegessen bzw. aus der Schale gelöffelt. Das Fruchtfleisch findet außerdem Verwendung als Zutat für Fruchtsalate oder Kaki-Mousse, als Füllung für Pfannkuchen oder als Beigabe zu Backwaren. Der Fruchtsaft ergibt ein wohlschmeckendes Getränk, das in Asien als Grundlage für Wein und Likör dient (Nowak \& Schulz 1998). Getrocknete Früchte sind als Kakifeigen vor allem in Ostasien ein beliebtes Trockenobst (BÄRTELS 1996).

Kakifrüchte sind reich an Zucker (15 bis $20 \%$ ) und somit ein guter Energiespender. Daneben sind sie ballaststoffreich und haben einen relativ hohen Nährwert, der etwa mit Weintrauben vergleichbar

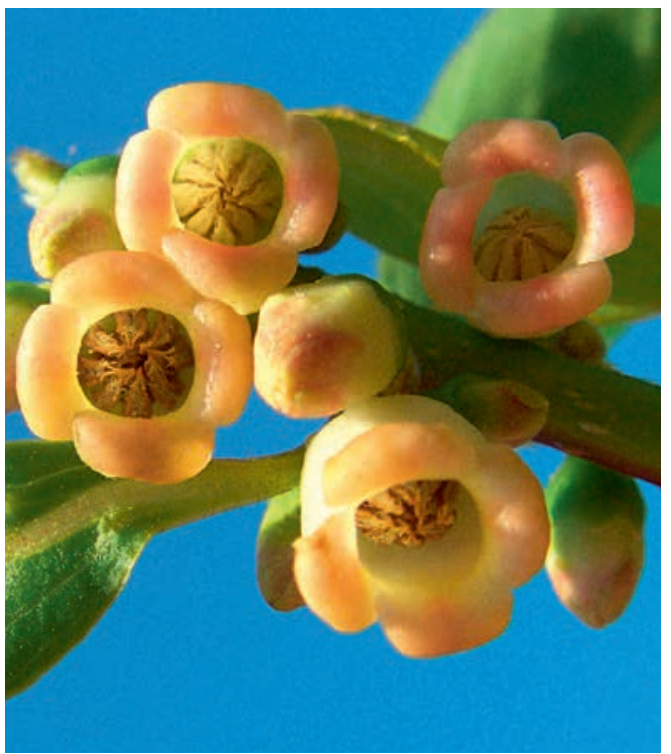

Abb. 14: Blüten von Diospyros lotus, Lotuspflaume, im Grugapark Essen. (Foto: A. JAGEL)

ist, einen geringen Fettanteil $(0,3 \%)$ und sind reich an Vitamin A, Vitaminen der B-Gruppe sowie an Vitamin C. Außerdem liefern sie Phosphor und Kalium und sind natriumarm (Trueb 1999).

Neben dem überwiegenden Anbau zur Nutzung der Früchte wird D. kaki auch als leicht zu überwinternde Zier- und Kübelpflanze geschätzt. Dabei wird sie vor allem wegen ihrer großen, glänzenden Blätter und der prächtigen Herbstfärbung kultiviert. Hierfür wurde sie schon 1796 nach England eingeführt (KeLly \& Hillier 2004). Das hell gemusterte, im Grundton schwarze Holz dient in Japan als Grundlage für feine Schnitzarbeiten und der Gerbstoff unreifer Früchte findet Verwendung in der Bearbeitung von Leder, zur Herstellung von Sake (japanischer Reiswein) und als Färbeoder Holzschutzmittel. Das Tannin soll außerdem als Feuchtigkeitscreme und Insektenmittel Anwendung finden (Lyle 2006). In der Volksmedizin werden Kakipflaumen zur Behandlung von Schilddrüsenerkrankungen verwendet (Trueb 1999). Die traditionelle chinesische Medizin nutzt einen Sud aus getrockneten Fruchtstielen und Kelchen gegen Husten und Atemnot 


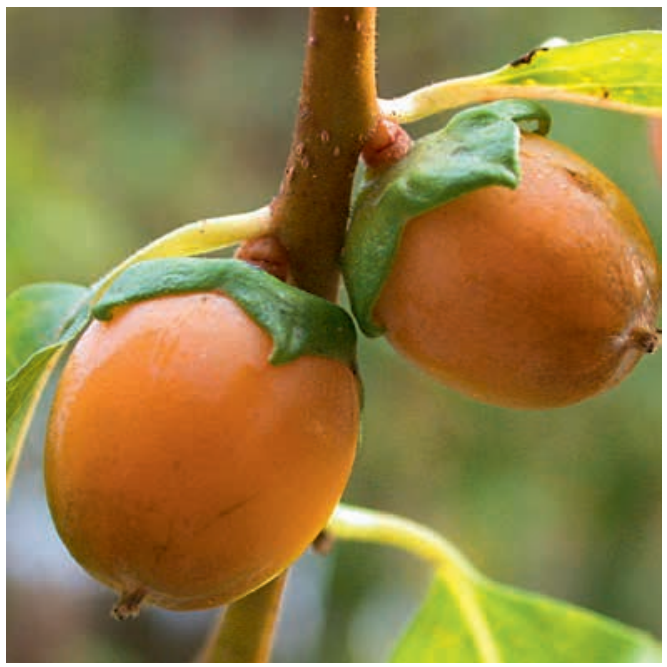

Abb. 15: Früchte von Diospyros lotus im Botanischen Garten Bochum. (Foto: A. Höggemeier)

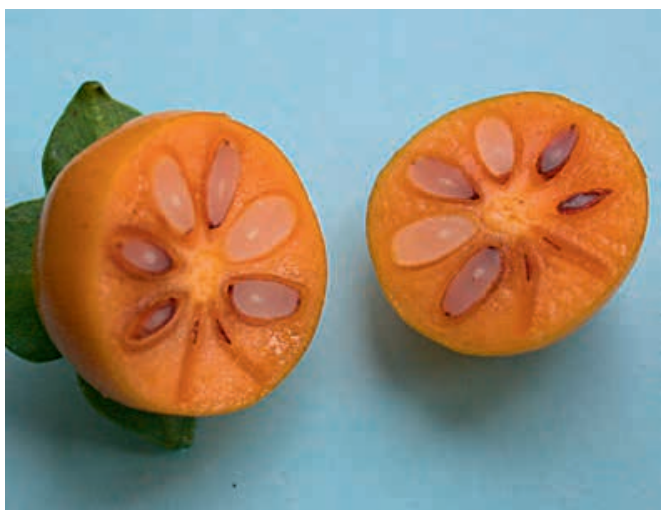

Abb. 16: Halbierte Frucht mit Samen von Diospyros lotus. (Foto: A. Höggemeier)
(NowaK \& Schulz 1998). Angeblich sollen die Früchte darüber hinaus eine Magen stärkende und bei Durchfallerkrankungen heilende sowie eine fieber- und blutdrucksenkende Wirkung haben.

\section{Diospyros lotus (Lotuspflaume) und Diospyros virginiana (Persimone)}

Lotuspflaumen (aus Ostasien) und Persimones (aus Nordamerika) werden bei uns fast ausschließlich in Botanischen Gärten und Sammlungen kultiviert, obwohl sie sogar winterhärter sind als D. kaki (Roloff \& BärTels 2006). Im subatlantischen Bochumer Botanischen Garten kommt die Lotuspflaume regelmäßig zur Blüte und bildet auch Früchte. Diese sind, wie auch die der Persimone, essbar, aber weitaus minderwertiger als die des Kakibaumes. Sie weisen einen vielfach höheren Tanningehalt auf und sind nur bei Vollreife oder nach Einwirkung von Frost genießbar (LyLE 2006) bzw. können mit heißem Wasser vorbehandelt werden (BRÜCHER 1977). Die Lotuspflaume kommt bei uns erst sehr spät zur Blüte, sodass auch die Früchte nur sehr selten vor dem Wintereinbruch vollständig ausreifen. Als Fruchtbaum sind sowohl Lotuspflaume als auch Persimone nur von lokaler Bedeutung und spielen für den weltweiten Handel keine Rolle (Trueb 1999). Ihre Früchte enthalten, wie auch die Wildform der Kaki, regelmäßig Samen.

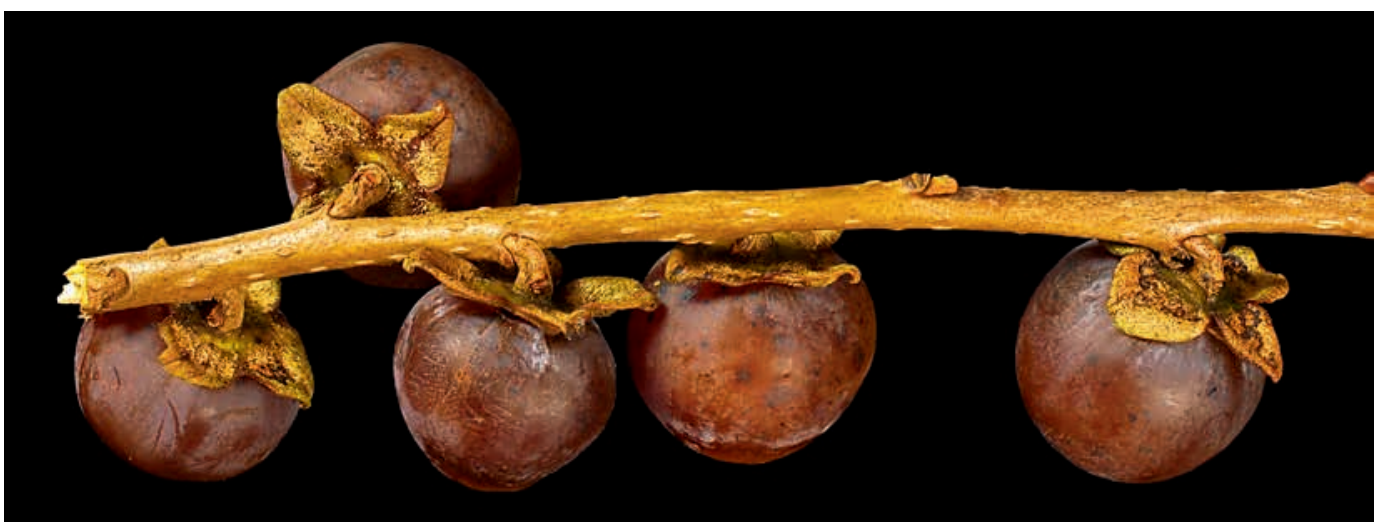

Abb. 17: Zweigstück mit Früchten von Diospyros lotus, Lotuspflaume, aus dem Botanischen Garten Frankfurt. (Foto: J. MarkWIRTH) 


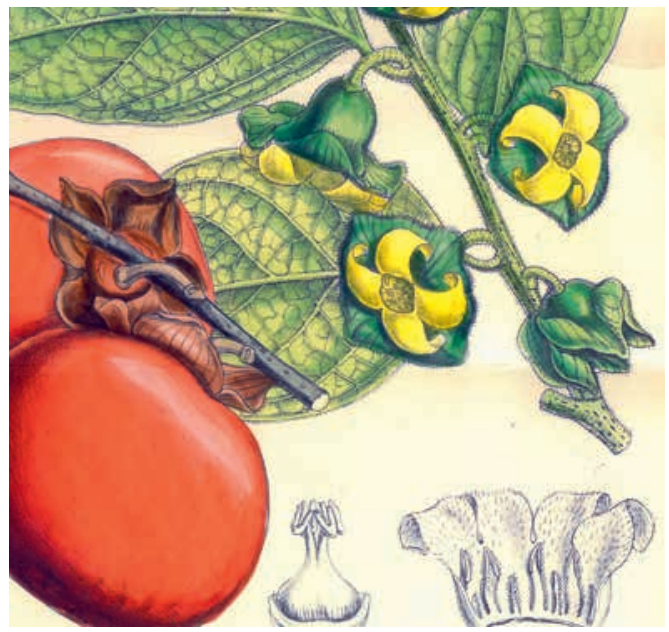

Diospyros-Pflanzen sind schon seit einer Weile in milden Gebieten Europas als Obstbäume beliebt. Diospyros kaki, abgebildet in Curtis's Botanical Magazine, Tab. 8127, (1907).

\section{Literatur}

BärTels, A, 1996: Farbatlas Tropenpflanzen. Zier- und Nutzpflanzen. - Stuttgart.

BrÜCHer, H. 1977: Tropische Nutzpflanzen. Ursprung, Evolution und Domestifikation. - Berlin.

Genaust, H. 2005: Etymologisches Wörterbuch der botanischen Pflanzennamen. 3. Aufl. - Hamburg.

Heinze, W. \& Schreiber, D. 1984: Eine neue Kartierung der Winterhärtezonen für Gehölze in Europa. - Mitt. Dtsch. Dendrol. Ges. 75: 11-56.

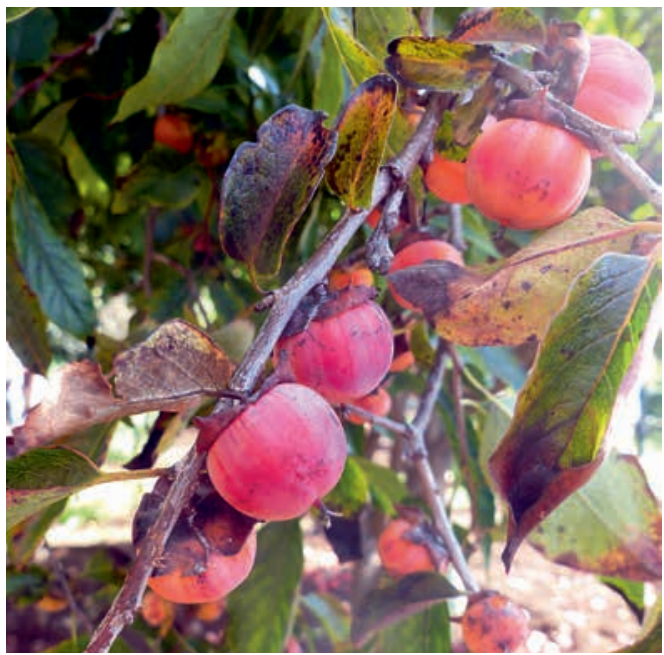

Abb. 19: In Gebieten mit milderem Klima als bei uns trifft man häufig auf Diospyros-Arten. Hier Diospyros lotus im Botanischen Garten Funchal, Madeira. (Foto: H. Steinecke)
Kelly, J. \& Hillier, J. (Hrsg.) 2004: The Hillier: Bäume \& Sträucher. 2. Aufl. - Braunschweig.

Lyle, S. 2006: Ultimate fruit \& nuts. A comprehensive guide to the cultivation, uses and health benefits of over 300 food-producing plants. - London.

NowaK, B, \& Schulz, B. 1998: Tropische Früchte. Biologie, Verwendung, Anbau und Ernte. - München.

Roloff, A. \& Bärtels, A. 2006: Flora der Gehölze. Bestimmung, Eigenschaften und Verwendung. 2. Aufl. - Stuttgart.

Trueb, L. F. 1999: Früchte und Nüsse aus aller Welt. - Stuttgart.

Wallnöfer, B. 2004: Ebenaceae. In Kubitzki, K. (Hrsg.): The families and genera of vascular plants, Vol. VI, Flowering plants, Dicotyledons, Celastrales, Oxalidales, Rosales, Cornales, Ericales, S. 125-139. - Berlin etc.

\section{Internetseiten}

http://www.obst-und-gemuese-aus-spanien.de/pdf/folder-kaki. pdf (Düsseldorf) [25.11.2010].

www.kaki-info.de.

\section{Anschriften der Autoren}

Dr. Ingo Hetzel, Idastr. 3, 45663 Recklinghausen, E-Mail: Ingo.Hetzel@botanik-bochum.de

Dr. Armin Jagel, Danziger Str. 2, 44789 Bochum, E-Mail: armin@jagel.nrw

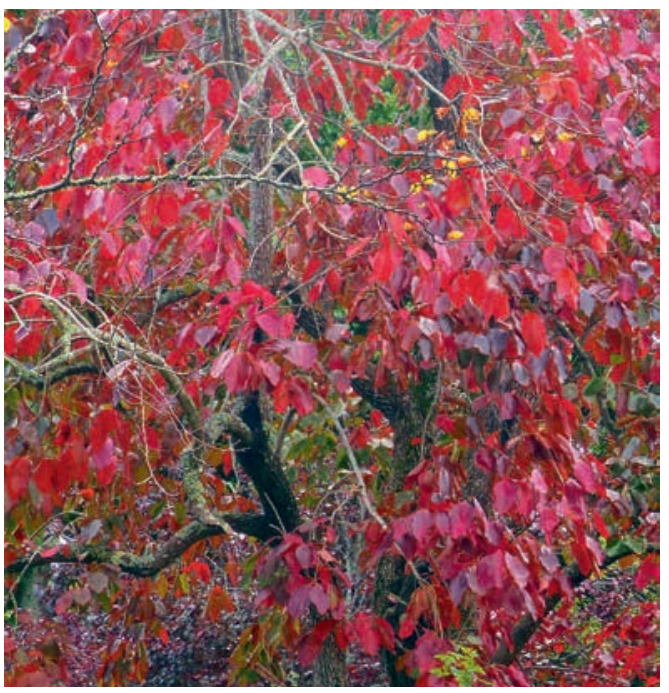

Abb. 20: Kakipflaume mit prächtigem Herbstlaub auf Madeira, November 2018. (Foto: H. Steinecke) 